\title{
Arithmetical properties of the values of functions satisfying certain functional equations of Poincaré
}

\author{
by \\ Masaaki Amou (Kiryu), Masanori Katsurada (Yokohama) \\ and KEIJO V̈̈̈̈NÄNEN (Oulu)
}

1. Introduction and the main result. Throughout this paper, let $K$ be an algebraic number field, $d$ its degree over the rational number field $\mathbb{Q}$, and $\mathcal{M}$ the set of all places of $K$. Let $q \in K$ and $v \in \mathcal{M}$ such that $|q|_{v}>1$. The completion of $K$ with respect to $v$ is denoted by $K_{v}$. Let $s$ be a positive integer, and $P(z)$ and $Q(z)$ polynomials in $K[z]$ satisfying

$$
P(0) \neq 0, \quad t:=\operatorname{deg} P \leq s, \quad u:=\operatorname{deg} Q .
$$

We consider the functional equation

$$
z^{s} f(z)=P(z) f(q z)+Q(z)
$$

which is a special case of the functional equations studied by Poincaré (see [14]). A solution of (1.2) on $K_{v}$ is given by

$$
f(z)=-\sum_{n=1}^{\infty} \prod_{i=1}^{n-1} \frac{\left(q^{-i} z\right)^{s}}{P\left(q^{-i} z\right)} \cdot \frac{Q\left(q^{-n} z\right)}{P\left(q^{-n} z\right)}
$$

which will be the object of this paper (see Subsection 1.2 for the details).

In the case where $K=\mathbb{Q}$ and $v=\infty$ (the unique infinite place), Duverney [7] proved the following irrationality result in an elegant way. Let $q$ be a rational integer with $|q|>1$. Assume that $f(z)$ in (1.3) is not a polynomial. Then $f(z)$ takes irrational values at all nonzero rational points except

2000 Mathematics Subject Classification: Primary 11J72; Secondary 11J82.

Key words and phrases: irrationality, irrationality measure, q-difference equation, $q$-hypergeometric series, Padé approximation, Siegel's lemma.

The first named author was supported in part by Grant-in-Aid for Scientific Research (No. 09640007), the Ministry of Education, Science, Sports and Culture of Japan. This research was partially done while he was visiting Department of Mathematics, the University of Oulu. He wishes to thank the department for their support and hospitality.

The second named author was supported in part by Grant-in-Aid for Scientific Research (No. 11640038), the Ministry of Education, Science, Sports and Culture of Japan. 
the poles of $f(z)$. For the proof he constructed auxiliary functions by using Siegel's lemma, and then iterated the functional equation appropriately many times to obtain good rational approximations of $f(\alpha)$. Similar methods have been applied to study Mahler functions (see e.g. Nishioka [13]).

The purpose of this paper is to extend and quantify Duverney's result in the general setting (as at the beginning of this section) under a certain condition on $q$ (Theorem 1 in Subsection 1.3). We further apply this result to investigating special values of fairly general $q$-hypergeometric series (Theorem 2 and its corollaries in Section 2). It is worthwhile mentioning that Theorem 2 covers many known irrationality results and contains new quantitative assertions. Indeed, Corollary 1 partly extends Stihl [18, Satz 1], while Corollary 3 quantifies a special case of Bézivin [1, Corollaire 1].

There are several works which deal with the arithmetical nature of solutions, not covered by (1.2), for the functional equations of Poincaré. We refer the reader to, e.g., Bundschuh and Waldschmidt [6], Duverney [8], MatalaAho [11], Matala-Aho and Väänänen [12], Stihl [17], Stihl and Wallisser [19], Töpfer [20], and Wallisser [24].

The plan of this paper is as follows. In Subsection 1.1 we recall the definition of the absolute height of algebraic numbers and the notion of the irrationality measure relative to $v$. In Subsection 1.2 we consider the functional equation (1.2) and obtain its solution (1.3). Our main result (Theorem 1) is presented in Subsection 1.3. In Section 2 we state and derive Theorem 2 as well as its corollaries. Theorem 1 is proved in the final section.

1.1. Notation. Let $K, \mathcal{M}, q$, and $v$ be as above. For any $w \in \mathcal{M}$, we normalize the absolute value $\mid{ }_{w}$ of $K$ so that $|p|_{w}=p^{-1}$ for a finite place $w$ lying over the prime number $p$ and $|x|_{w}=|x|(x \in \mathbb{Q})$ for an infinite place $w$, where || denotes the ordinary absolute value. We define the function $\delta(w)$ on $\mathcal{M}$ to be $\delta(w)=0$ or 1 according as $w$ is a finite or an infinite place.

The product formula

$$
\prod_{w}|\alpha|_{w}^{d_{w}}=1
$$

holds for all nonzero $\alpha \in K$, where $d_{w}=\left[K_{w}: \mathbb{Q}_{w}\right]$. The absolute height $h(\alpha)$ of $\alpha \in K$ is defined by

$$
h(\alpha)=\prod_{w} \max \left(1,|\alpha|_{w}^{d_{w} / d}\right) .
$$

Then, for any nonzero elements $\alpha$ and $\beta$ of $K$, we have

$$
h\left(\alpha^{-1}\right)=h(\alpha), \quad h(\alpha \beta) \leq h(\alpha) h(\beta) .
$$

The absolute height for the vector $\underline{\alpha}=\left(\alpha_{1}, \ldots, \alpha_{k}\right), \alpha_{i} \in K$, is defined by

$$
h(\underline{\alpha})=\prod_{w} \max \left(1,|\underline{\alpha}|_{w}^{d_{w} / d}\right)
$$


with

$$
|\underline{\alpha}|_{w}=\max \left(\left|\alpha_{1}\right|_{w}, \ldots,\left|\alpha_{k}\right|_{w}\right) .
$$

For any nonzero vector $\underline{\alpha}$, the fundamental inequality

$$
h(\underline{\alpha})^{-d / d_{v}} \leq|\underline{\alpha}|_{v} \leq h(\underline{\alpha})^{d / d_{v}}
$$

holds. In fact, the second inequality follows from the definition of $h(\underline{\alpha})$, while the first follows from the product formula, on writing $|\underline{\alpha}|_{v}=\left|\alpha_{i}\right|_{v}$ with some $i$ :

$$
|\underline{\alpha}|_{v}=\left|\alpha_{i}\right|_{v}=\left(\prod_{w \neq v}\left|\alpha_{i}\right|_{w}^{d_{w} / d}\right)^{-d / d_{v}} \geq\left(\prod_{w} \max \left(1,|\underline{\alpha}|_{w}^{d_{w} / d}\right)\right)^{-d / d_{v}} .
$$

We introduce the quantity $\lambda$ defined by

$$
\lambda=\frac{d \log h(q)}{d_{v} \log |q|_{v}} .
$$

If the estimate $h(\underline{\alpha}) \leq h(q)^{c}$ holds with some positive number $c$, then the fundamental inequality gives

$$
|q|_{v}^{-c \lambda} \leq|\underline{\alpha}|_{v} \leq|q|_{v}^{c \lambda}
$$

Inequalities of this type will be used in Section 3.

REMARK. We always have $\lambda \geq 1$, and $\lambda=1$ if and only if $|q|_{w} \leq 1$ for all $w \neq v$. In particular, if $K$ is the rational or an imaginary quadratic number field, $v=\infty$, and $q$ is an algebraic integer in $K$, then we have $\lambda=1$.

For $\omega \in K_{v} \backslash K$, the irrationality measure relative to $v$ of $\omega$ is defined to be the infimum of the positive numbers $\mu$ for which the inequality

$$
|\omega-\theta|_{v}>h(\theta)^{-\mu}
$$

is satisfied for any $\theta \in K$ with sufficiently large $h(\theta)$, and it is denoted by $\mu(\omega ; K, v)$. For $\omega \in \mathbb{R} \backslash \mathbb{Q}$, in particular, $\mu(\omega ; \mathbb{Q}, \infty)$ is nothing but the ordinary irrationality measure of $\omega$.

The use of the absolute height makes it possible to consider simultaneously the numbers of $K_{v}$ for every $v$. It also enables us to apply Siegel's lemma in the form given by Bombieri [2] (see Subsection 3.2). This lemma indeed gives a better measure than that derived from the original Siegel lemma, even in the case where $K=\mathbb{Q}$ and $v=\infty$.

1.2. Solution of the functional equation. In this subsection we first show that (1.2) has a unique solution in the formal power series ring $K[[z]]$, which converges in a neighbourhood of the origin. Then we will see that the solution (1.3) is obtained by extending this power series to the whole $K_{v}$ using (1.2). Though the procedure above was essentially given in Duverney [7], we describe the details for our later purpose. 
Let us write the polynomials in (1.2) as

$$
P(z)=\sum_{i=0}^{t} \alpha_{i} z^{i}, \quad Q(z)=\sum_{i=0}^{u} \beta_{i} z^{i}
$$

Then the power series

$$
f(z)=\sum_{n=0}^{\infty} f_{n} z^{n}
$$

satisfies (1.2) if and only if

$$
f_{n-s}-\sum_{i=0}^{t} \alpha_{i} q^{n-i} f_{n-i}-\beta_{n}=0
$$

for all $n \geq 0$, where we use the convention that $f_{i}=0$ for $i<0$ and $\beta_{n}=0$ for $n>u$. This is a system of linear equations for $f_{n}$, which can be solved uniquely in $K$ by the recursion formula

$$
f_{n}=\frac{1}{\alpha_{0} q^{n}}\left(f_{n-s}-\sum_{i=1}^{t} \alpha_{i} q^{n-i} f_{n-i}-\beta_{n}\right) .
$$

Hence (1.2) has a unique solution (1.5) in $K[[z]]$. We claim that the power series converges in a neighbourhood of the origin. In fact, it follows from (1.6) with $|q|_{v}>1$ that

$$
\left|f_{n}\right|_{v} \leq(t+2)^{\delta(v)}\left|\alpha_{0}^{-1} \underline{\xi}\right|_{v} \max \left(1,\left|f_{0}\right|_{v}, \ldots,\left|f_{n-1}\right|_{v}\right)
$$

for all $n \geq 0$, where

$$
\underline{\xi}=\left(1, \alpha_{0}, \ldots, \alpha_{t}, \beta_{0}, \ldots, \beta_{u}\right) .
$$

This implies that

$$
\left|f_{n}\right|_{v} \leq(t+2)^{\delta(v) n}\left|\alpha_{0}^{-1} \underline{\xi}\right|_{v}^{n+1},
$$

by which our claim holds. We denote by $\mathbf{D}$ the disk in $K_{v}$ (with center at $z=0$ ) where (1.5) converges. The power series (1.5) is continued, beyond $\mathbf{D}$, to the whole $K_{v}$ by using (1.2) repeatedly, and the resulting function is again denoted by $f(z)$. It remains to prove that $f(z)$ coincides with (1.3). Let $\mathcal{P}$ be the set defined by

$$
\mathcal{P}=\left\{q^{k} \beta \mid P(\beta)=0, k \geq 1\right\} .
$$

Then, by iterating (1.2),

$$
f(z)=\prod_{i=1}^{k} \frac{\left(q^{-i} z\right)^{s}}{P\left(q^{-i} z\right)} \cdot f\left(q^{-k} z\right)-\sum_{i=1}^{k} \prod_{j=1}^{i-1} \frac{\left(q^{-j} z\right)^{s}}{P\left(q^{-j} z\right)} \cdot \frac{Q\left(q^{-i} z\right)}{P\left(q^{-i} z\right)}
$$


for any $z \in K_{v} \backslash \mathcal{P}$ provided that $q^{-k} z \in \mathbf{D}$. Since the first product on the right-hand side tends to zero as $k \rightarrow \infty$, (1.3) holds for any $z \in K_{v} \backslash \mathcal{P}$, as desired.

Let $\mathcal{P}_{0}$ be the set of all elements $\alpha \in \mathcal{P}$ at which $f(z)$ does not have a limit. It follows from the functional equation (1.2) that $q^{k} \beta \in \mathcal{P}_{0}$ implies $q^{n} \beta \in \mathcal{P}_{0}$ for all $n \geq k$. This shows that $\mathcal{P}_{0}$ is either empty or an infinite set. Hence $f(z)$ can be a rational function if and only if it is a polynomial.

1.3. Statement of the main result. The following theorem is the main result of this paper, which extends and quantifies the result of Duverney [7, Théorème 2].

THEOREM 1. Let $f(z)$ be the function given in (1.3) with $P(z), Q(z)$ $\in K[z]$ satisfying (1.1). Assume that $f(z)$ is not a polynomial, and that there exist positive numbers $\eta, 1<\eta<2$, and $\varrho$ for which

$$
\lambda<\Theta(\eta, \varrho):=\frac{\Lambda+\varrho(\eta-1)}{\Lambda+\widetilde{\eta}},
$$

where $\lambda$ is the quantity defined by (1.4) and

$$
\Lambda=\Lambda(\eta, \varrho)=(2-\eta+\varrho)\{1+(2-\eta+\varrho) s / 2\}, \quad \widetilde{\eta}=\frac{\eta^{3}}{6(2-\eta)} .
$$

Then, for any nonzero $\alpha \in K \backslash \mathcal{P}_{0}, f(\alpha)$ does not belong to $K$. Furthermore,

$$
\mu(f(\alpha) ; K, v) \leq \frac{d}{d_{v}} \inf \frac{\varrho(\eta-1)+\Lambda}{\varrho(\eta-1)-(\lambda-1) \Lambda-\lambda \widetilde{\eta}},
$$

where the infimum is taken over all positive numbers $\eta, 1<\eta<2$, and $\varrho$ for which (1.10) holds. In particular, $f(\alpha)$ is not a Liouville number.

Let $\lambda(s)=\sup \Theta(\eta, \varrho)$, where the supremum is taken over all positive numbers $\eta, 1<\eta<2$, and $\varrho$. Note that $\lambda(s)$ depends only on $s$. The following table provides fairly sharp lower bounds for $\lambda(s)$ with $1 \leq s \leq 5$.

\begin{tabular}{cccccc}
\hline$s$ & 1 & 2 & 3 & 4 & 5 \\
$\lambda(s)>$ & 1.028 & 1.014 & 1.010 & 1.007 & 1.006 \\
\hline
\end{tabular}

If $\lambda=1$, then for any positive number $\eta, 1<\eta<2$, the condition (1.10) is valid for all sufficiently large positive numbers $\varrho$. Hence we have the following corollary to Theorem 1.

Corollary. Let $f(z)$ be as above. Assume that $f(z)$ is not a polynomial, and that $\lambda=1$. Then, for any nonzero $\alpha \in K \backslash \mathcal{P}_{0}, f(\alpha)$ does not belong to $K$. Furthermore,

$$
\mu(f(\alpha) ; K, v) \leq \frac{d}{d_{v}} \inf \frac{\varrho(\eta-1)+\Lambda}{\varrho(\eta-1)-\widetilde{\eta}},
$$


where the infimum is taken over all positive numbers $\eta, 1<\eta<2$, and $\varrho$ for which $\varrho(\eta-1)-\widetilde{\eta}>0$. In particular, $f(\alpha)$ is not a Liouville number.

Besides the classical case (where $K$ is the rational or an imaginary quadratic number field, $v=\infty$, and $q$ is an algebraic integer in $K$ ), our corollary applies for example if $K=\mathbb{Q}, q=p^{-k}$ with some positive integer $k$ and ||$_{v}=||_{p}$, or more generally if $q$ is any PV-number.

Let $\mu(s)=\inf (\varrho(\eta-1)+\Lambda) /(\varrho(\eta-1)-\widetilde{\eta})$, where the infimum is taken over all positive numbers $\eta, 1<\eta<2$, and $\varrho$ for which $\varrho(\eta-1)-\widetilde{\eta}>0$. Note that $\mu(s)$ depends only on $s$. Since the function $(\varrho(\eta-1)+\Lambda) /(\varrho(\eta-1)-\widetilde{\eta})$ attains the value $(76+169 s) / 15$ at $(\eta, \varrho)=(3 / 2,6)$, we have

$$
\mu(s) \leq \frac{76+169 s}{15} .
$$

The following table provides fairly sharp upper bounds for $\mu(s)$ with $1 \leq$ $s \leq 5$.

\begin{tabular}{cccccc}
\hline$s$ & 1 & 2 & 3 & 4 & 5 \\
$\mu(s)<$ & 15.61 & 26.57 & 37.40 & 48.19 & 58.96 \\
\hline
\end{tabular}

When $s=1, P(z) \equiv 1$, and $Q(z) \equiv-1$, the functional equation (1.2) is reduced to

$$
z f(z)=f(q z)-1
$$

and the solution (1.3) to

$$
T_{q}(z)=\sum_{n=0}^{\infty} \frac{z^{n}}{q^{\left(\begin{array}{c}
n+1 \\
2
\end{array}\right)}},
$$

which is called the Tschakaloff function. The study on the arithmetical nature of the values of $T_{q}(z)$ goes back to Tschakaloff [21] in 1921, and subsequent research has been made by many authors. Concerning our main theorem, we quote the result which asserts that, for any nonzero $\alpha \in K$,

$$
\mu\left(T_{q}(\alpha) ; K, v\right) \leq \frac{d}{d_{v}} \frac{1+\sqrt{5}}{1+\sqrt{5}-2 \lambda}
$$

under the assumption

$$
\lambda<\frac{1+\sqrt{5}}{2} .
$$

When $K$ is an imaginary quadratic number field and $v=\infty$, Bundschuh [4] first obtained the result above, which is better than the assertion of Theorem 1 in this particular case. Then it was proved in the general setting by Väänänen [23]. The result of Bundschuh was extended by Stihl [17], which quantifies Stihl and Wallisser [19, Satz 2]. He considered the functional equation of the type

$$
f(q z)=a_{0}\left(z-a_{1}\right) f(z)+Q(z),
$$


where $q, a_{0}$, and $a_{1}$ are elements of an imaginary quadratic number field $K$, and $Q(z) \in K[z]$. Then, in the case of $a_{1}=0$, his result gives the same conclusion as in [4].

2. Application to $q$-hypergeometric series. In this section we apply Theorem 1 to the function $\phi(z)=\phi(z ; s, P)$ on $K_{v}$ defined by

$$
\phi(z)=\sum_{k=0}^{\infty} \frac{q^{-s\left(\begin{array}{c}
k \\
2
\end{array}\right)}}{P(1) P\left(q^{-1}\right) \ldots P\left(q^{-(k-1)}\right)} z^{k},
$$

where $s$ is a positive integer, and $P(z)$ is a polynomial in $K[z]$ satisfying (1.1) and

$$
P\left(q^{-k}\right) \neq 0 \quad \text { for } k=0,1,2, \ldots
$$

By introducing the reciprocal polynomial $\widehat{P}(z)=z^{t} P(1 / z)$, the definition (2.1) can be rewritten as

$$
\phi(z)=\sum_{k=0}^{\infty} \frac{q^{-(s-t)\left(\begin{array}{c}
k \\
2
\end{array}\right)}}{\widehat{P}(1) \widehat{P}(q) \ldots \widehat{P}\left(q^{k-1}\right)} z^{k} .
$$

We note that $\phi(z)$ is an entire function on $K_{v}$. If $P(z)$ in $K[z]$ has a factorization of the form

$$
P(z)=\left(1-a_{1} z\right) \ldots\left(1-a_{t} z\right),
$$

then $\phi(z)$ can be expressed in terms of a $q$-hypergeometric function ${ }_{1} \phi_{s}$, namely

$$
\begin{aligned}
{ }_{1} \phi_{s}\left(q^{-1} ; a_{1}, \ldots, a_{t},\right. & \underbrace{0, \ldots, 0}_{s-t} ; q^{-1}, z) \\
& =\sum_{k=0}^{\infty} \frac{\left(q^{-1} ; q^{-1}\right)_{k}}{\left(a_{1} ; q^{-1}\right)_{k} \ldots\left(a_{t} ; q^{-1}\right)_{k}\left(q^{-1} ; q^{-1}\right)_{k}} q^{-s\left(\begin{array}{l}
k \\
2
\end{array}\right) z^{k},}
\end{aligned}
$$

where $(x ; y)_{0}=1$ and

$$
(x ; y)_{k}=(1-x)(1-x y) \ldots\left(1-x y^{k-1}\right)
$$

for $k \geq 1$ (see Stihl [18]).

We now consider the connection between $\phi(z)$ and the solution of the functional equation (1.2). For any nonzero $\alpha \in K$, we define the function $f(z ; \alpha)$ on $K_{v}$ by

$$
f(z ; \alpha)=\sum_{k=0}^{\infty} \frac{q^{-s\left(\begin{array}{c}
k+1 \\
2
\end{array}\right)} z^{s k}}{P\left(q^{-1} z\right) \ldots P\left(q^{-k} z\right)} \alpha^{k},
$$

which satisfies

$$
\alpha z^{s} f(z)=P(z) f(q z)-P(z)
$$


with $f(z)=f(z ; \alpha)$, and

$$
\phi(\alpha)=f(q ; \alpha) .
$$

The functional equation (1.2) becomes (2.5) by replacing $P(z)$ with $\alpha^{-1} P(z)$, and $Q(z)$ with $-\alpha^{-1} P(z)$, respectively, and the solution (1.3) becomes (2.4) by the same replacement.

Definition. We define the set $\mathcal{E}(s, P)$ associated with $s$ and $P(z)$ to be the set consisting of $\alpha \in K$ for which $f(z ; \alpha)$ is a polynomial.

If (1.10) holds for some positive numbers $\eta, 1<\eta<2$, and $\varrho$, it follows from Theorem 1 that, for any nonzero $\alpha \in K, f(q ; \alpha)$ belongs to $K$ if and only if $\alpha$ belongs to $\mathcal{E}(s, P)$. Thus, in view of $(2.6)$, we have

TheOrem 2. Let $\phi(z)=\phi(z ; s, P)$ be the function defined by (2.1) with $P(z) \in K[z]$ satisfying (1.1) and (2.2). Assume that there exist positive numbers $\eta, 1<\eta<2$, and $\varrho$ for which (1.10) holds. Then, for nonzero $\alpha \in K, \phi(\alpha)$ belongs to $K$ if and only if $\alpha$ belongs to $\mathcal{E}(s, P)$. Furthermore, for any nonzero $\alpha \in K$, not an element of $\mathcal{E}(s, P)$, the inequality (1.12) holds with $\phi(\alpha)$ instead of $f(\alpha)$.

REMARK. We see from $(2.5)$ that $f(z ; \alpha)$ with $\alpha \neq 0$ is not a polynomial when $t=\operatorname{deg} P<s$. Hence $\mathcal{E}(s, P)=\{0\}$ in this case. On the other hand, in the case $t=s$, we claim that

$$
\mathcal{E}(s, P) \subset\left\{a_{s} q^{k} \mid k \geq 1\right\},
$$

where $a_{s}$ is the coefficient of the leading term of $P(z)$. For the proof, we assume that $f(z ; \alpha)$ is a polynomial. Note first that $f(z ; \alpha)$ with $\alpha \neq 0$ is not a constant; otherwise $f(z ; \alpha) \equiv 1$, and so $\alpha z^{s}=0$ by $(2.5)$, which implies $\alpha=0$. Comparing the leading terms of both sides of (2.5), we find that $\alpha=a_{s} q^{k}$, where $k$ is the degree of $f(z ; \alpha)$. This shows (2.7) as desired.

We give several corollaries to Theorem 2. The following corollary in the case $t<s$ is a direct consequence of the first part of the remark above.

COROLlary 1. Under the notation and the assumptions of Theorem 2, assume further that $t<s$. Then, for any nonzero $\alpha \in K, \phi(\alpha)$ does not belong to $K$, and the inequality (1.12) holds with $\phi(\alpha)$ instead of $f(\alpha)$.

This corollary partly extends the main result of Stihl [18] (see also Katsurada [9]). Though he proved in fact the linear independence of function values, we quote here the result by restricting it to the irrationality case. Let $s$ be a positive integer, $K$ an imaginary quadratic number field, $v=\infty$, and $P(z)$ a polynomial in $K[z]$ of the form

$$
P(z)=\left(1-q^{-\nu_{1}} z\right) \ldots\left(1-q^{-\nu_{t_{0}}} z\right)\left(1-b_{t_{0}+1} z\right) \ldots\left(1-b_{t} z\right),
$$


where $0 \leq t_{0} \leq t<s, \nu_{i} \in \mathbb{N}$, and $b_{j} \in K \backslash\{0\}$ satisfy $b_{j} \neq q^{k}$ for all integers $k \geq 0$. Then, for any nonzero $\alpha \in K$, it follows that

$$
\mu(\phi(\alpha ; s, P) ; K, v) \leq \frac{\gamma}{\gamma-\lambda(\gamma-1)}
$$

under the condition

$$
\lambda<\frac{\gamma}{\gamma-1}
$$

where

$$
\gamma=\frac{s}{2(s-t)^{2}}\left(\sqrt{\gamma_{0}}+(s-t)(1+2 s)+t^{2}-t_{0}\right)
$$

with

$$
\gamma_{0}=\left(s+t_{0}-t(1+t)\right)^{2}+4(s-t)(1+s)\left(t^{2}-t_{0}\right)+4(s-t)^{2} s^{2} .
$$

The result above is better than the assertion of Corollary 1 in this particular case. For the proof Stihl constructed Padé approximations for $\phi(z)$, where the functional equation

$$
\left\{P(q \Delta)-z \Delta^{s}\right\} \phi(z)=P(q)
$$

plays a central role. Here $\Delta$ is the $q^{-1}$-difference operator acting as $(\Delta \phi)(z)$ $=\phi\left(q^{-1} z\right)$.

The following two corollaries deal with the case where $s=t$. We note that the qualitative part of these results were obtained by Bézivin [1], in the case where $K$ is an imaginary quadratic number field and $v=\infty$.

COROLlary 2. Let the notations and the assumptions be as in Theorem 2 with $s=1$ and $P(z)=A z+B, A B \neq 0$. Then, for nonzero $\alpha \in K$, $\phi(\alpha)$ belongs to $K$ if and only if $\alpha$ is of the form $\alpha=A q^{k}$ with some integer $k \geq 1$. Moreover, for any $\alpha \in K$ satisfying $\alpha \neq A q^{k}$ for all integers $k \geq 1$, the inequality (1.12) holds with $\phi(\alpha)$ instead of $f(\alpha)$.

Remark. The same assertion as in Corollary 2 holds for $\phi(z ; s, P)$ defined with a slightly general $P(z)=A z^{s}+B$, where $s$ is any positive integer. In fact, the general case is reduced to the case $s=1$ by replacing $q$ with $q^{s}$, while the corresponding $\lambda$ in (1.4) is unchanged upon this replacement.

Proof of Corollary 2. Our task is to show that

$$
\mathcal{E}(1, P) \backslash\{0\}=\left\{A q^{k} \mid k \geq 1\right\} .
$$

For any nonzero $\alpha \in K$, let $f(z)=f(z ; \alpha)$ be the function given by (2.4) with $s=1$ and $P(z)=A z+B$. Substituting (1.5) into both sides of (2.5) and equating the coefficients, we obtain $f_{0}=1, f_{1}=(B q)^{-1} \alpha$, and

$$
f_{n}=\left(B q^{n}\right)^{-1}\left(\alpha-A q^{n-1}\right) f_{n-1} \quad \text { for } n \geq 2 .
$$

This clearly implies the desired assertion. Thus the corollary is proved. 
REMARK. Under the assumption in Corollary 2, by (2.8) we have

$$
f\left(z ; A q^{k}\right)=1+\sum_{n=1}^{k} f_{k, n} z^{n}
$$

for any positive integer $k$, where

$$
f_{k, n}=(A / B)^{n} q^{k-\left(\begin{array}{c}
k+1 \\
2
\end{array}\right)} \prod_{i=1}^{n-1}\left(q^{k}-q^{i}\right) .
$$

In view of $(2.3)$, we see that $\phi(z ; 1, q-z)$ is the $q$-exponential function

$$
E_{q}(z)=\sum_{n=0}^{\infty} \frac{z^{n}}{(q-1)\left(q^{2}-1\right) \ldots\left(q^{n}-1\right)},
$$

which satisfies the functional equation

$$
E_{q}(q z)=(1+z) E_{q}(z) .
$$

The study on the arithmetical nature of $E_{q}(z)$ goes back to Lototsky [10] in 1943, and subsequent research has been made by various authors. Concerning Corollary 2 above, we quote the result which asserts that, for any nonzero $\alpha \in K$ with $\alpha \neq-q^{k}(k=1,2, \ldots)$,

$$
\mu\left(E_{q}(\alpha) ; K, v\right) \leq \frac{d}{d_{v}} \frac{7}{7-4 \lambda}
$$

under the condition $\lambda<7 / 4$. Bundschuh [3] first obtained the result when $K$ is an imaginary quadratic number field and $v=\infty$. Then the same assertion was proved for general $K$ with an infinite place $v$ of $K$ by Popov [15], and in full generality by Väänänen [22]. The result of Stihl [17] mentioned at the end of Subsection 1.3 generalizes Bundschuh's result above. Moreover, it gives a better measure for $\phi(\alpha)$ than that given in Corollary 2 in this particular case. To see this we note that $\phi(z)$ in Corollary 2 is a solution of the functional equation (1.14) with $a_{0}=q / B, a_{1}=A$, and $Q(z)=1+(q A) / B$. Then Stihl's result implies the same conclusion as Bundschuh's result if $(q A) / B=-q^{k}$ for some integer $k$, while if $(q A) / B \neq-q^{k}$ for any integer $k$, with the condition $\lambda<\sqrt{2}$, it also yields

$$
\mu(\phi(\alpha) ; K, v) \leq \frac{2}{2-\sqrt{2} \lambda} .
$$

We state the last corollary which is a direct consequence of Theorem 2 with (2.7).

COROLlary 3. Let the notation and assumptions be as in Theorem 2 with $P(z)=\left(z-a_{1}\right) \ldots\left(z-a_{s}\right)$ for nonzero elements $a_{i}$ of $K$. Then, for any nonzero $\alpha \in K$ satisfying $\alpha \neq q^{k}(k=1,2, \ldots), \phi(\alpha)$ does not belong to $K$, and the inequality (1.12) holds with $\phi(\alpha)$ instead of $f(\alpha)$. 
We conclude this section by giving an example pertaining to Corollary 3 in the case where $K=\mathbb{Q}$ and $v=\infty$. Let $q$ be a positive integer with $|q|>1$, and set

$$
P(z)=(z-q)\left(z-q^{\nu+1}\right)
$$

with a nonnegative integer $\nu$. From $(2.3)$ the function $\phi(z)=\phi(z ; 2, P)$ (defined with $P(z)$ ) on $\mathbb{R}$ is

$$
\phi(z)=\sum_{n=0}^{\infty} \frac{z^{n}}{(q ; q)_{n}\left(q^{\nu+1} ; q\right)_{n}} .
$$

We set $\mathcal{K}_{q, \nu}(z)=\phi\left(-z^{2} / 4\right)$. This is a $q$-analogue of the Siegel $E$-function

$$
\mathcal{K}_{\nu}(z)=\sum_{n=0}^{\infty} \frac{\left(-z^{2} / 4\right)^{n}}{n !(\nu+1) \ldots(\nu+n)},
$$

whose arithmetical properties were studied by Siegel [16]. Let $\alpha$ be a nonzero rational number. Since $-\alpha^{2} / 4$ does not belong to the set $\left\{q^{k} \mid k \geq 1\right\}$, it follows from Corollary 3 that $\mathcal{K}_{q, \nu}(\alpha)$ is an irrational number and not a Liouville number.

3. Proof of the main theorem. In this section we prove Theorem 1. Let $f(z)$ be the function given by (1.3) with $P(z), Q(z) \in K[z]$ satisfying (1.1), which coincides with the power series (1.5) on D. Assume that $f(z)$ is not a polynomial. As was remarked in Subsection 1.2, this means that it is not a rational function. Before going into the precise steps of the proof, we claim that it suffices to prove Theorem 1 in the case where $\alpha \in \mathbf{D}$. To show this we assume that the assertion of Theorem 1 holds for all nonzero $\alpha \in K \cap \mathbf{D}$. Let $\alpha^{\prime}$ be an arbitrary nonzero element of $K \backslash \mathcal{P}_{0}$, and let $k$ be the least positive integer satisfying $q^{-k} \alpha^{\prime} \in \mathbf{D}$. Then for $\alpha=q^{-k} \alpha^{\prime}$ we see that $f(\alpha)$ does not belong to $K$ and (1.12) holds. An equivalent form of (1.9) is

$$
f\left(q^{-k} z\right)=\prod_{i=1}^{k} \frac{P\left(q^{-i} z\right)}{\left(q^{-i} z\right)^{s}} \cdot f(z)+\sum_{i=1}^{k} \prod_{j=i+1}^{k} \frac{P\left(q^{-j} z\right)}{\left(q^{-j} z\right)^{s}} \cdot \frac{Q\left(q^{-i} z\right)}{\left(q^{-i} z\right)^{s}}
$$

in which we set $z=\alpha^{\prime}$. This shows

$$
\prod_{i=1}^{k} P\left(q^{-i} \alpha^{\prime}\right) \neq 0
$$

otherwise $f(\alpha)$ does belong to $K$ in view of (3.1), which is a contradiction. Therefore $f\left(\alpha^{\prime}\right)$ can be written as

$$
f\left(\alpha^{\prime}\right)=X f(\alpha)+Y
$$


with some elements $X \neq 0$ and $Y$ of $K$. This implies that $f\left(\alpha^{\prime}\right)$ does not belong to $K$ and the inequality (1.12) holds with $f\left(\alpha^{\prime}\right)$ instead of $f(\alpha)$, as required.

3.1. Main proposition. Throughout the following sections let $\alpha$ be any nonzero element of $K$. We will prove that $f(\alpha)$ does not belong to $K$ and the inequality (1.12) holds. From the reduction argument above, we may assume that $\alpha \in \mathbf{D}$. Since in what follows we consider the values of $f(z)$ only at the points $z=q^{-k} \alpha(k=0,1,2, \ldots)$, we treat the function $f(z)$ expressed as in (1.5). We denote by $v_{0}$ an arbitrarily chosen element of $\mathcal{M}$ which is different from $v$. The constants in $O$-notation may depend on $P, Q, K, v, v_{0}, \alpha, \eta$, and $\varrho$, but not on $r$.

We now state the main proposition which can be used to prove Theorem 1 .

Proposition. Let $\eta, 1<\eta<2$, and $\varrho$ be positive numbers, and let $\Lambda=\Lambda(\eta, \varrho)$ and $\widetilde{\eta}$ be the quantities defined in (1.11). Then, for any positive integer $r$, there exist two linearly independent linear forms

$$
L_{i, r}=U_{i, r} f(\alpha)+V_{i, r}, \quad U_{i, r}, V_{i, r} \in K \quad(i=1,2)
$$

such that for $i=1,2$ we have

$$
\begin{aligned}
& \max \left(\left|U_{i, r}\right|_{w},\left|V_{i, r}\right|_{w}\right) \\
& \quad \leq 2^{\delta(w) O(r)} \max \left(1,|q|_{w}\right)^{\Lambda r^{2}+O(r)}|\underline{\xi}|_{w}^{O(r)} \max \left(1,|\alpha|_{w}\right)^{O(r)}
\end{aligned}
$$

for all $w \in \mathcal{M}$ with $w \neq v_{0}$, and

$$
\begin{aligned}
& \max \left(\left|U_{i, r}\right|_{v_{0}},\left|V_{i, r}\right|_{v_{0}}\right) \\
& \leq 2^{O(r)} \max \left(1,|q|_{v_{0}}\right)^{\Lambda r^{2}+O(r)} h(q)^{\left(d / d_{v_{0}}\right) \widetilde{\eta} r^{2}}|\underline{\xi}|_{v_{0}}^{O(r)} \max \left(1,|\alpha|_{v_{0}}\right)^{O(r),}
\end{aligned}
$$

where $\underline{\xi}$ is the vector given by (1.7), and

$$
\left|L_{i, r}\right|_{v} \leq|q|_{v}^{-\varrho(\eta-1) r^{2}+O(r)} .
$$

To see how Theorem 1 follows from the Proposition we define

$$
L=f(\alpha)-\theta, \quad \theta \in K .
$$

By the Proposition, there exists a linear form $L_{r}=U_{r} f(\alpha)+V_{r}\left(L_{1, r}\right.$ or $\left.L_{2, r}\right)$ such that

$$
\Delta=\left|\begin{array}{cc}
1 & -\theta \\
U_{r} & V_{r}
\end{array}\right| \neq 0
$$

Note that $\Delta=L_{r}-U_{r} L$. We now choose $v_{0} \neq v$ and proceed in almost the same way as in the proof of the case $\Delta \neq 0$ in Bundschuh and Väänänen [5], pp. 190-192. This gives, under the assumption $\Lambda+\varrho(\eta-1)-\lambda \Lambda-\lambda \widetilde{\eta}>0$,

$$
|L|_{v}>H^{-\frac{d}{d v} \frac{\Lambda+\varrho(\eta-1)}{\Lambda+\varrho(\eta-1)-\lambda \Lambda-\lambda \tilde{\eta}}-\frac{O(1)}{\sqrt{\log H}}}
$$


where $H=\max \left(h(\theta), H_{0}\right)$ with some positive constant $H_{0}$. Thus Theorem 1 holds. Our task is therefore reduced to proving the Proposition, which will be done in the following subsections. We give here an outline.

In the next subsection we construct an auxiliary function of the form

$$
L(z)=A(z) f(z)+B(z), \quad A(z), B(z) \in K[z]
$$

such that the degrees of $A(z)$ and $B(z)$ are at most $r$, and ord $L(z) \geq \eta r$ (see Lemma 1), where ord $L(z)$ is the order of the zeros of $L(z)$ at $z=0$. Our construction depends on Siegel's lemma in the form given by Bombieri [2], which ensures a sharp upper bound of $h(\underline{x})$, where $\underline{x}$ is the vector whose elements are the coefficients of $A(z)$ and $B(z)$. It also ensures an additional property that $|\underline{x}|_{w} \leq 1$ for all $w \in \mathcal{M}$ different from $v_{0}$.

In Subsection 3.3 we introduce

$$
\widetilde{L}(z):=P(z) L(q z)=\widetilde{A}(z) f(z)+\widetilde{B}(z),
$$

say, and prove that the resultant $\mathcal{R}(z)$ of $L$ and $\widetilde{L}$ with respect to $f(z)$ does not vanish. This gives a sharp upper bound of ord $L(z)$, and enables us to show the existence of a positive integer $k$ in a certain range such that $\mathcal{R}\left(q^{-k} \alpha\right) \neq 0$ (see Lemma 2 ).

In Subsection 3.4 we consider the relation between $f(\alpha)$ and $f\left(q^{-k} \alpha\right)$ (see Lemma 3). In Subsection 3.5, by combining the conclusions of Subsections 3.2-3.4, the desired linear forms are constructed from $L\left(q^{-k} \alpha\right)$ and $\widetilde{L}\left(q^{-k} \alpha\right)$ with certain multipliers.

It is of interest to note that we follow closely the ideas of Siegel's method.

3.2. Construction of auxiliary functions. We construct auxiliary functions stated in Lemma 1 below. To this aim we employ Siegel's lemma in the form given by Bombieri [2]:

Siegel's lemma (Bombieri [2]). Let $\gamma=4 d^{2 d}\left|D_{K}\right|^{1 / 2}$, where $D_{K}$ is the discriminant of $K$. Let $M$ and $N$ be positive integers with $M<N$. Then there is a nontrivial solution $\underline{x}=\left(x_{1}, \ldots, x_{N}\right) \in K^{N}$ of

$$
\sum_{j=1}^{N} a_{i j} x_{j}=0 \quad(i=1, \ldots, M)
$$

with

$$
h(\underline{x}) \leq \gamma(2 N \gamma)^{M /(N-M)}\left(\prod_{i=1}^{M} \prod_{w} \max _{1 \leq j \leq N}\left|a_{i j}\right|_{w}^{d_{w} / d}\right)^{1 /(N-M)} .
$$

Moreover, given any place $v^{\prime}$ of $K$, we may find such a solution with the additional property

$$
|\underline{x}|_{w} \leq 1 \quad \text { for all } w \neq v^{\prime} .
$$


Lemma 1. For any positive number $\eta, 0<\eta<2$, and any positive integer $r$ there exist auxiliary polynomials

$$
A(z)=\sum_{i=0}^{r} a_{i} z^{i}, \quad B(z)=\sum_{i=0}^{r} b_{i} z^{i},
$$

not both zero, with coefficients $a_{i}, b_{i} \in K$, such that

$$
\sigma:=\operatorname{ord} L(z) \geq \eta r
$$

where

$$
L(z)=A(z) f(z)+B(z),
$$

and the following estimates are valid:

$$
\begin{gathered}
h(\underline{x}) \leq 2^{O(r)} h(q)^{\tilde{\eta} r^{2}}, \quad \underline{x}=\left(a_{0}, \ldots, a_{r}, b_{0}, \ldots, b_{r}\right), \\
|\underline{x}|_{w} \leq 1 \quad \text { for all } w \neq v_{0}, \\
|L(z)|_{v} \leq(r+2)^{\delta(v)} c^{\sigma+1}|z|_{v}^{\sigma} \quad \text { for }|z|_{v} \leq 1 /(2 c),
\end{gathered}
$$

where $c=(t+2)^{\delta(v)}\left|\alpha_{0}^{-1} \underline{\xi}\right|_{v}$ with $\underline{\xi}$ given by $(1.7)$.

Proof. We have

$$
A(z) f(z)+B(z)=\sum_{n=0}^{\infty} C_{n} z^{n}
$$

where

$$
C_{n}=\sum_{i=0}^{r} a_{i} f_{n-i}+b_{n}
$$

with the conventions $b_{n}=0$ for $n>r$ and $f_{i}=0$ for $i<0$. To satisfy the inequality (3.5) we need

$$
C_{n}=0 \quad \text { for } n=0,1,2, \ldots,[\eta r] .
$$

This is a system of $[\eta r]+1$ linear homogeneous equations with $2 r+2$ unknowns $a_{i}, b_{i}$. We claim that, for any nonnegative integer $n$,

$$
\prod_{w} \max _{0 \leq i \leq n}\left|f_{i}\right|_{w}^{d_{w} / d} \leq c_{0}^{n} h(q)^{\left(\begin{array}{c}
n+1 \\
2
\end{array}\right)}
$$

with a positive constant $c_{0}$ depending only on $P$ and $Q$. In fact, it follows from (1.6) that

$$
\left|f_{n}\right|_{w} \leq(t+2)^{\delta(w)}\left|\alpha_{0}^{-1} \underline{\xi}\right|_{w} \max \left(1,|q|_{w}^{-1}\right)^{n} \max \left(1,\left|f_{0}\right|_{w}, \ldots,\left|f_{n-1}\right|_{w}\right)
$$

for all $w \in \mathcal{M}$. Hence

$$
\left|f_{n}\right|_{w} \leq(t+2)^{\delta(w) n}\left|\alpha_{0}^{-1} \underline{\xi}\right|_{w}^{n+1} \max \left(1,|q|_{w}^{-1}\right)^{\left(\begin{array}{c}
n+1 \\
2
\end{array}\right)},
$$


which implies the desired inequality. Then, by applying Siegel's lemma above, there exists a nontrivial solution $\underline{x}$ such that (3.7) holds and

$$
h(\underline{x}) \leq 2^{O(r)}\left(\prod_{n=0}^{[\eta r]} c_{0}^{n} h(q){ }^{\left(\begin{array}{c}
n+1 \\
2
\end{array}\right)}\right)^{1 /(2 r+2-([\eta r]+1))} .
$$

Since the exponent of $h(q)$ on the right-hand side is bounded from above by

$$
\frac{1}{(2-\eta) r} \sum_{n=0}^{[\eta r]}\left(\begin{array}{c}
n+1 \\
2
\end{array}\right)=\widetilde{\eta} r^{2}+O(r),
$$

(3.6) holds.

To prove (3.8) we denote the right-hand side of (3.9) by $z^{\sigma} E(z)$, where

$$
E(z)=\sum_{n=0}^{\infty} C_{n+\sigma} z^{n} .
$$

Since

$$
\left|C_{n+\sigma}\right|_{v} \leq(r+2)^{\delta(v)} \max \left(1,\left|f_{0}\right|_{v}, \ldots,\left|f_{n+\sigma}\right|_{v}\right) \leq(r+2)^{\delta(v)} c^{n+1+\sigma}
$$

by (1.8), the inequality (3.8) holds. Thus the lemma is proved.

3.3. Application of resultant. We prove Lemma 2 below which gives nonvanishing of a certain determinant and its consequences. Let $A(z)$ and $B(z)$ be polynomials in $K[z]$, and set

$$
L(z)=A(z) f(z)+B(z) .
$$

By the functional equation (1.2) we obtain

$$
\widetilde{L}(z):=P(z) L(q z)=\widetilde{A}(z) f(z)+\widetilde{B}(z),
$$

where

$$
\widetilde{A}(z)=z^{s} A(q z), \quad \widetilde{B}(z)=P(z) B(q z)-Q(z) A(q z) .
$$

We denote by $\mathcal{R}(z)$ the resultant of $L$ and $\widetilde{L}$ with respect to $f(z)$, that is,

$$
\mathcal{R}(z)=\left|\begin{array}{ll}
A(z) & B(z) \\
\widetilde{A}(z) & \widetilde{B}(z)
\end{array}\right| .
$$

Lemma 2. Let $A(z)$ and $B(z)$ be polynomials in $K[z]$, not both zero, of degree at most $r$. Then the resultant $\mathcal{R}(z)$ does not vanish, and this implies that

$$
\text { ord } L(z) \leq 2 r+\max (s, u)
$$

and for any positive real $\varrho$ there exists a positive integer $k=k(r)$ satisfying

$$
\varrho r \leq k \leq(2-\eta+\varrho) r+\max (s, u)
$$

such that

$$
\mathcal{R}\left(q^{-k} \alpha\right) \neq 0
$$


Proof. Assume to the contrary that $\mathcal{R}(z)$ vanishes. Then, using

$$
\mathcal{R}(z)=A(z) \widetilde{L}(z)-\widetilde{A}(z) L(z),
$$

we obtain

$$
P(z) A(z) L(q z)=z^{s} A(q z) L(z) .
$$

Comparing the orders of the zeros at $z=0$ of both sides, we see that their difference is $s>0$, and this implies a contradiction.

The upper bound for ord $L(z)$ follows from ord $L(z) \leq \operatorname{deg} \mathcal{R}$ and

$$
\operatorname{deg} \mathcal{R} \leq \max (\operatorname{deg} A(z) \widetilde{B}(z), \operatorname{deg} \widetilde{A}(z) B(z)) \leq 2 r+\max (s, u) .
$$

The existence of $k$ in the assertion is a direct consequence of the upper bound above for the degree of the nonzero polynomial $\mathcal{R}(z)$.

3.4. Iteration of the functional equation. We show a relation between $f(\alpha)$ and $f\left(q^{-k} \alpha\right)$ for any positive integer $k$.

Lemma 3. For any positive integer $k$, we have

$$
q^{u k} z^{s k} f\left(q^{-k} z\right)=M_{k}(z, q) f(z)+N_{k}(z, q)
$$

with some polynomials $M_{k}(z, q)$ and $N_{k}(z, q)$ in $K[z, q]$. Moreover,

$$
\begin{aligned}
\max & \left(\left|M_{k}(\alpha, q)\right|_{w},\left|N_{k}(\alpha, q)\right|_{w}\right) \\
& \leq 2^{\delta(w) O(k)} \max \left(1,|q|_{w}\right)^{s k^{2} / 2+O(k)}|\underline{\xi}|_{w}^{O(k)} \max \left(1,|\alpha|_{w}\right)^{O(k)}
\end{aligned}
$$

for all $w \in \mathcal{M}$.

Proof. It follows from (3.1) that (3.14) holds with

$$
M_{k}(z, q)=q^{s\left(\begin{array}{c}
k+1 \\
2
\end{array}\right)+u k} \prod_{i=1}^{k} P\left(q^{-i} z\right)
$$

and

$$
N_{k}(z, q)=\sum_{i=1}^{k} q^{s\left\{\left(\begin{array}{c}
k+1 \\
2
\end{array}\right)-\left(\begin{array}{c}
i \\
2
\end{array}\right)\right\}+u k} z^{s(i-1)} \prod_{j=i+1}^{k} P\left(q^{-j} z\right) \cdot Q\left(q^{-i} z\right),
$$

both belonging to $K[z, q]$. Since

$$
\begin{aligned}
& \max \left(\operatorname{deg}_{z} M_{k}, \operatorname{deg}_{z} N_{k}\right) \leq s k+u, \\
& \max \left(\operatorname{deg}_{q} M_{k}, \operatorname{deg}_{q} N_{k}\right) \leq s\left(\begin{array}{c}
k+1 \\
2
\end{array}\right)+u k,
\end{aligned}
$$

(3.15) holds.

3.5. Proof of the Proposition. Let $\eta, 1<\eta<2$, and $\varrho$ be positive numbers, and $r$ a positive integer. Let $A(z)$ and $B(z)$ be polynomials in $K[z]$, not both zero, of degree at most $r$, which satisfy all the conditions in Lemma 1. We choose a $k$ satisfying (3.11) and (3.12). Note that $k=O(r)$. 
We consider the quantities

$$
L_{r}=q^{r k} q^{u k} \alpha^{s k} L\left(q^{-k} \alpha\right), \quad \widetilde{L}_{r}=q^{(r+\max (s, u)) k} q^{u k} \alpha^{s k} \widetilde{L}\left(q^{-k} \alpha\right) .
$$

By (3.14) we may write

$$
L_{r}=U f(\alpha)+V, \quad \widetilde{L}_{r}=\widetilde{U} f(\alpha)+\widetilde{V},
$$

where

$$
U=q^{r k} M_{k}(\alpha, q) A\left(q^{-k} \alpha\right), \quad V=q^{r k} N_{k}(\alpha, q) A\left(q^{-k} \alpha\right)+q^{r k} q^{u k} \alpha^{s k} B\left(q^{-k} \alpha\right)
$$

and $\widetilde{U}, \widetilde{V}$ have the corresponding expressions with $q^{(r+\max (s, u)) k}$ replaced by $q^{r k}$ and $\widetilde{A}, \widetilde{B}$ by $A, B$. Then we show that the pair $\left(L_{1, r}, L_{2, r}\right)=\left(L_{r}, \widetilde{L}_{r}\right)$ satisfies the conditions stated in the Proposition. Since

$$
\left|\begin{array}{ll}
U & V \\
\widetilde{U} & \widetilde{V}
\end{array}\right|=q^{(2 r+\max (s, u)) k} q^{u k} \alpha^{s k} M_{k}(\alpha, q) \mathcal{R}\left(q^{-k} \alpha\right) \neq 0
$$

by an elementary operation on the determinant, $L_{r}$ and $\widetilde{L}_{r}$ are linearly independent linear forms of 1 and $f(\alpha)$.

We next show that $L_{r}$ and $\widetilde{L}_{r}$ also satisfy other conditions in the Proposition. Consider $L_{r}$ (the consideration of $\widetilde{L}_{r}$ is analogous). It follows from (3.15) that

$$
\begin{aligned}
& \max \left(|U|_{w},|V|_{w}\right) \\
& \quad \leq 2^{\delta(w) O(r)}|\underline{x}|_{w} \max \left(1,|q|_{w}\right)^{r k+s k^{2} / 2+O(r)}|\underline{\xi}|_{w}^{O(r)} \max \left(1,|\alpha|_{w}\right)^{O(r)}
\end{aligned}
$$

for all $w$. We have

$$
|\underline{x}|_{v_{0}} \leq 2^{O(r)} h(q)^{\left(d / d_{v_{0}}\right) \tilde{\eta} r^{2}}
$$

by (3.6) and $|\underline{x}|_{w} \leq 1$ for all $w \neq v_{0}$ by (3.7). We also have

$$
r k+s k^{2} / 2 \leq(2-\eta+\varrho)\{1+(2-\eta+\varrho) s / 2\} r^{2}+O(r)
$$

by (3.11), and the main term of the right-hand side is $\Lambda r^{2}$ by (1.11). Hence, combining these estimates, we obtain (3.2) and (3.3).

For an upper bound of $\left|L_{r}\right|_{v}$, we have

$$
\left|L_{r}\right|_{v} \leq 2^{O(r)}|q|_{v}^{r k} c^{\sigma}\left|q^{-k} \alpha\right|_{v}^{\sigma}
$$

by (3.8). Since $\eta r \leq \sigma \leq 2 r+\max (s, u)$ by (3.5) and Lemma 2 , this inequality implies that

$$
\left|L_{r}\right|_{v} \leq 2^{O(r)}|q|_{v}^{-r k(\eta-1)} .
$$

Hence (3.4) follows from (3.11). This completes the proof of the Proposition, and hence that of Theorem 1.

Acknowledgements. The authors would like to express their sincere gratitude to Professor Daniel Duverney, who kindly pointed out that Theorem 1 in the original form could be improved by a refined use of (3.12). 
According to his suggestions the estimate for the irrationality measure was sharpened to the present form.

\section{References}

[1] J.-P. Bézivin, Indépendance linéaire des valeurs des solutions transcendantes de certaines équations fonctionnelles, Manuscripta Math. 6 (1988), 103-129.

[2] E. Bombieri, On G-functions, in: Recent Progress in Analytic Number Theory, Vol. 2, Academic Press, 1981, 1-68.

[3] P. Bundschuh, Arithmetische Untersuchungen unendlicher Produkte, Invent. Math. 6 (1969), 275-295.

[4] —, Verschärfung eines arithmetischen Satzes von Tschakaloff, Portugal. Math. 33 (1974), 1-17.

[5] P. Bundschuh and K. Väänänen, Arithmetical investigations of a certain infinite product, Compositio Math. 91 (1994), 175-199.

[6] P. Bundschuh and M. Waldschmidt, Irrationality results for theta functions by Gel'fond-Schneider's method, Acta Arith. 53 (1989), 289-307.

[7] D. Duverney, Propriétés arithmétiques des solutions de certaines équations fonctionnelles de Poincaré, J. Théor. Nombres Bordeaux 8 (1996), 443-447.

[8] - Formes linéaires d'approximation et irrationalité de valeurs de q-fonctions, Portugal. Math. 56 (1999), 205-220.

[9] M. Katsurada, Linear independence measures for values of Heine series, Math. Ann. 284 (1989), 449-460.

[10] A. V. Lototsky, Sur l'irrationalité d'un produit infini, Mat. Sb. 12 (54) (1943), 262-272.

[11] T. Matala-Aho, Remarks on the arithmetic properties of certain hypergeometric series of Gauss and Heine, Acta Univ. Oulu. Ser. A Sci. Rerum Natur. 219 (1991), $1-112$.

[12] T. Matala-Aho and K. Väänänen, On Diophantine approximations of Mock theta functions of third order, Ramanujan J. 4 (2000), 13-28.

[13] K. Nishioka, Mahler Functions and Transcendence, Lecture Notes in Math. 1631, Springer, 1996.

[14] H. Poincaré, Sur une classe nouvelle de transcendantes uniformes, Journal de Math. (4) 6 (1890), 313-365; Oeuvres, tome IV, 537-582.

[15] A. Yu. Popov, Approximation of values of some infinite products, Vestnik Moskov. Univ. Ser. A 45 (1990), 3-6 (in Russian); English transl.: Moscow Univ. Math. Bull. 45 (1990), 4-6.

[16] C. L. Siegel, Transcendental Numbers, Ann. of Math. Stud. 16, Princeton Univ. Press, 1949.

[17] T. Stihl, Irrationalitätsmaße für Werte der Lösungen einer Funktionalgleichung von Poincaré, Arch. Math. (Basel) 41 (1983), 531-537.

[18] —, Arithmetische Eigenschaften spezieller Heinescher Reihen, Math. Ann. 268 (1984), 21-41.

[19] T. Stihl und R. Wallisser, Zur Irrationalität und linearen Unabhängigkeit der Werte der Lösungen einer Funktionalgleichung von Poincaré, J. Reine Angew. Math. 341 (1983), 98-110.

[20] T. Töpfer, Arithmetical properties of functions satisfying q-difference equations, Analysis 15 (1995), 25-49. 
[21] L. Tschakaloff, Arithmetische Eigenschaften der unendlichen Reihe $\sum_{\nu=0}^{\infty} x^{\nu}$ $\times a^{-\nu(\nu-1) / 2}$, I, Math. Ann. 80 (1921), 62-74; II, ibid. 84 (1921), 100-114.

[22] K. Väänänen, On the approximation of certain infinite product, Math. Scand. 73 (1993), 197-208.

[23] — A linear independence measure for the values of Tschakaloff function at algebraic points, Math. Univ. Oulu, preprint, February, 1993, 10 pages.

[24] R. Wallisser, Über die arithmetische Natur der Werte der Lösungen einer Funktionalgleichung von H. Poincaré, Acta Arith. 25 (1973), 81-92.

Department of Mathematics

Gunma University

Tenjin-cho 1-5-1

Kiryu 376-8515, Japan

E-mail: amou@math.sci.gunma-u.ac.jp

Department of Mathematics

University of Oulu

P.O. Box 3000

90014 Oulu, Finland

E-mail: kvaanane@sun3.oulu.fi
Mathematics, Hiyoshi Campus

Keio University

Hiyoshi 4-1-1, Kouhoku-ku

Yokohama 223-8521, Japan E-mail: masanori@math.hc.keio.ac.jp

Received on 26.6.2000

and in revised form on 23.11.2000 